\section{La enseñanza de la Medicina en la facultad de Albacete.}

\author{
Sr. Editor:
}

La Memoria para la creación de la Facultad de Medicina en la Universidad de Castilla-La Mancha ${ }^{1}$ establecía en su capítulo 7, sobre Planificación Educativa, la puesta en marcha de "un modelo de aprendizaje no repetitivo de los existentes, sustituyendo el sistema de enseñanza 'tradicional' por un modelo de enseñanza integrada...". Al mismo tiempo, abogaba por que los futuros médicos alcanzaran "una experiencia clínica adecuada, llevada a cabo tanto en hospitales como en instituciones extrahospitalarias". El objetivo era "incluir todos los recursos sanitarios de la comunidad, y no solo los hospitales". Y lo justificaba de forma precisa al señalar: "Un peligro de los centros universitarios es el de que, por sus características, concentran patología muy especializada, que origina una deformación en el estudiante, con lo cual la adquisición de las habilidades clínicas básicas puede ponerse en peligro. Este problema se pretende subsanar en el proyecto que ocupa a la UCLM mediante la integración real y efectiva de los Centros de Atención Primaria en la enseñanza de la Medicina".

Estos planteamientos vanguardistas permitieron que la Facultad de Medicina de Albacete fuera la primera de España en que se cursó una asignatura obligatoria de Atención Primaria, habiendo llegado a contar hasta con cinco profesores asociados. Sin embargo, la obligada adaptación al Espacio Europeo de Educación Superior (más conocido como Plan Bolonia) supuso la elaboración de un plan de estudios que ponía en peligro el futuro de la enseñanza de la Medicina de Familia en las Facultades de Medicina de la Universidad de Castilla-La Mancha. Dentro de la Facultad de Medicina de Albacete (en ese momento la de Ciudad Real aún no había empezado a funcionar) se gestaron una serie de alianzas para hacerse con el control de los órganos directivos y la comisión encargada de elaborar el nuevo plan de estudios. Aunque se partía de unas premisas básicas de una Orden Ministerial2 ${ }^{2}$, daban suficiente margen como para hacer un jugoso reparto de créditos, lo que supone disponibilidad de recursos económicos, de profesorado..., y, en definitiva, poder dentro del sistema. Los directivos de la facultad pretendieron que la Medicina de Familia quedase como algo testimonial en una asignatura que se pretendió llamar Geriatría y Medicina de Familia. En aquel momento fue decisiva la movilización de la mayoría de los entonces profesores asociados de atención primaria (cuatro de los cinco), médicos de familia de formación MIR, con una larga trayectoria de implicación en la docencia. Finalmente, una vez agotadas las posibilidades de negociación dentro de la Facultad de Medicina, tuvimos que recurrir al papel mediador del entonces Consejero de Sanidad, Fernando Lamata. Así se pudo conseguir una asignatura de Medicina de Familia: 6 créditos (el mínimo indispensable para tener una asignatura) en $5^{\circ}$ curso, además de un rotatorio obligatorio en $6^{\circ}$.

Como resultado de aquel conflicto, el equipo directivo de la facultad emprendió una serie de medidas para dejar el control del área en manos del profesor que no se había implicado en la lucha por conseguir la asignatura independiente, persona acomodaticia a sus intereses, llamativamente sin formación específica en Medicina de Familia (es decir, sin formación MIR...). Durante el curso 2014-2015 ha sido el único profesor asociado de Medicina de Familia, para cuyo nombramiento se permitieron, sin ningún rubor, vulnerar las bases de la convocatoria, que exigía que los candidatos ocuparan una plaza asistencial en alguno de los centros de salud docentes y estar ejerciendo una actividad asistencial en ese momento (no olvidemos que son plazas de Profesor Asociado Clínico, que tienen como objetivo poner en contacto a los alumnos con profesionales que atienden pacientes, en este caso en Atención Primaria). El Subdirector Médico de la Gerencia de Atención Integrada, que fue el seleccionado por la UCLM, ocupa un despacho en el Hospital General y obviamente no realiza actividad asistencial. De todas formas no ha estado solo, ya que tranquilamente han nombrado "a criterio" a otros "profesores", a pesar de haber quedado un candidato en reserva.

El día 6 de mayo se convocaron cinco nuevas plazas de profesor asociado de Medicina de Familia para la Facultad de Albacete. Insuficientes, porque a la asignatura que se imparte en $5^{\circ}$ curso hay que añadir el rotatorio que se va a realizar en centros de salud en $6^{\circ}$, pero algo es algo. Sin embargo hay 
que lamentar que las plazas vienen asignadas a centros de salud específicos, con lo que se libran de presencias molestas, como los Médicos de Familia del centro de salud Zona IV. ¡Qué curioso que uno de los centros de salud que más se ha implicado en la docencia tanto de pregrado como de postgrado sea descartado en esta convocatoria!

Pero incluso más grave es que al mismo tiempo convoquen una plaza de mejora a la que solo puede presentarse el único profesor asociado de Medicina de Familia que existe en estos momentos, el que ni tiene formación MIR, ni cumplía los criterios de selección cuando le dieron la plaza ni los cumple ahora.

Es bien conocida la trayectoria de esta persona, y son muchas las sombras que se atribuyen a sus actividades, especialmente en el ámbito de la investigación. Recientemente advertí al Rector de la UCLM, aportando pruebas, de uno de los aspectos sobre los que existen en abundancia, que demuestran la vulneración de unos de los principios éticos fundamentales en publicación científica. Se trata de la práctica de publicaciones múltiples, ya denunciada en un informe del Centre de Recerca en Economía i Salud (CRES) de la Universidad Pompeu Fabra ${ }^{3}$ (ver páginas 43 y 95). Lejos de tomar medidas para reconducir esta lamentable situación, se le premia con la convocatoria de una plaza "personalizada" de mejora.

En definitiva, una historia de despropósitos, de posiciones de poder por parte de personas de dudosa competencia y de venganzas personales.

\section{BIBLIOGRAFÍA}

1. Memoria para la creación de la Facultad de Medicina en la Universidad de Castilla-La Mancha. Albacete: Universidad de Castilla-La Mancha; 1998.

2. ORDEN ECI/332/2008, de 13 de febrero, por la que se establecen los requisitos para la verificación de los títulos universitarios oficiales que habiliten para el ejercicio de la profesión de Médico. BOE. 2008;40: 8351-5.

3. Peiró S, Sanfélix Gimeno G, Ridao López M, Cervera Casino P. Revisión sistemática de evaluaciones económicas de las estatinas comercializadas en España. Disponible en: http://catsalut.gencat.cat/web/.content/minisite/catsalut/ proveidors_professionals/medicaments_farmacia/ farmaeconomica/caeip/informes_dictaments/estatines/ estatinas.informe.es.caeip.2007.pdf. [Consultado el 8-52015].

Francisco Escobar Rabadán

Médico de Familia del Centro de Salud Zona IV de Albacete. 